\title{
La gestion des risques, questions et réponses
}

\author{
par Jean-Claude Deutsch
}

CERGRENE

\section{INTRODUCTION}

L'assainissement, et en particulier l'assainissement des eaux pluviales a été, depuis le début des temps historiques, une technique liée à l'urbanisme. Les premières cités, au troisième millénaire avant J.C., possédaient des équipements de stockage des eaux de ruissellement qui, vraisemblablement, étaient réutilisées par la suite.

Ce lien n'a fait que se renforcer au cours du temps. La mise en place d'une civilisation en grande partie urbaine sur l'ensemble de la planète a déjà débuté et devrait se généraliser dans le courant du XXI ${ }^{e}$ siècle d'après les spécialistes de prospective les plus réputés. Elle va amplifier les problèmes posés par le cycle urbain de l'eau.

Dès aujourd'hui, on peut constater que la population urbaine en France représente près de $80 \%$ de la population totale. C'est un chiffre que l'on retrouve plus ou moins dans les pays développés. Dans les pays en développement, le taux de progression de cette population urbaine peut atteindre des valeurs impressionnantes, jusqu'à $7 \%$ l'an pour certaines villes.

Dans le tableau 1 est présenté l'exemple de Sao-Paulo au Brésil. On notera que l'augmentation de la population reste encore très forte jusqu'au début du prochain siècle. Les chiffres indiqués pour l'année 2005, sont évidemment des estimations.

Les conséquences de l'artificialisation du cadre de vie sur le cycle de l'eau sont bien connues: inondations de plus en plus fréquentes et aggravation de la pollution du milieu naturel sont les plus connues et aussi les plus redoutées.

La réflexion qui a été menée, en particulier par les scientifiques, pour lutter contre ces deux types de catastrophe a conduit au concept de gestion du risque. Celui-ci est considéré comme étant la conjonction d'un aléa et d'une vulnérabilité.

Cette conceptualisation a été plus ou moins largement acceptée par l'ensemble des acteurs sociaux concernés ces dernières années. Nous allons l'utiliser comme grille de lecture pour essayer dans un premier temps de caractériser l'état des connaissances que l'on possède aujourd'hui dans le domaine de la lutte contre les inondations d'une part, et dans le domaine de la protection de l'environnement contre les pollutions d'autre part. Nous donnerons ensuite les éléments qui nous paraissent indispensables à la mise en place d'une gestion simultanée des deux risques en essayant d'être le plus réaliste possible. Enfin, l'approche scientifique et technique sera relativisée par rapport à d'autres approches.

\section{Risk management, questions and answers}

The development of urbanisation has leaded, concerning urban storm drainage control, to the concept of risk management. This concept is considered, classically, as the conjonction of a hazard and a vulnerability.

The analysis of the flooding risk and of the pollution risk show a lack of knowledge for the estimation of the hazard and some difficulties to define the vulnerability.

Nevertherless the management of risk must deal with the present level of knowledge, the regulation context, and decision making process between the different institutions and between the different geographic levels. To achieve this management, a panel of approaches can be used : scientific and technic, formation and sensibilisation of elected people, cultural, regulational and application of the precautionary principle. But it seems, in the end, that the risk management is essentially a political affair. 
Tableau 1. - Croissance de la population et de la superficie urbanisée à Sao-Paolo (Brésil).

\begin{tabular}{|c|c|c|c|c|}
\hline Année & Population & $\begin{array}{c}\text { Surface } \\
\text { urbanisée }\end{array}$ & \\
\hline & $\begin{array}{c}\text { Millions } \\
\text { d'habitants }\end{array}$ & $\begin{array}{c}\text { Taux } \\
\text { d'augmen- } \\
\text { tation }\end{array}$ & $\begin{array}{c}\text { Par rapport } \\
\text { au bassin } \\
\text { versant (\%) }\end{array}$ & $\begin{array}{c}\text { Taux } \\
\text { d'augmen- } \\
\text { tation }\end{array}$ \\
\hline 1905 & 0,3 & 267 & 0,6 & 433 \\
\hline 1930 & 1,1 & 218 & 3,2 & 172 \\
\hline 1954 & 3,5 & 166 & 8,7 & 154 \\
\hline 1973 & 9,3 & 59 & 22,1 & 63 \\
\hline 1985 & 14,8 & 72 & 36,0 & 30 \\
\hline 2005 & 25,5 & & 46,8 & \\
\hline
\end{tabular}

\section{II — LES INONDATIONS}

Le phénomène considéré à l'origine de l'aléa est soit l'événement pluvieux, soit la crue dans les réseaux hydrographiques naturels ou artificiels. L'aléa considéré est une notion statistique : la période de retour du phénomène considéré. Ainsi on parle couramment en hydrologie urbaine de la pluie décennale, et en hydrologie générale de la crue millénale, voire décamillénale. Cette définition est généralement difficilement compréhensible pour des non-scientifiques car elle heurte le sens commun. En effet, comment accepter qu'un événement, disons vingtennal, puisse se produire à un mois d'intervalle comme cela arrive parfois. Au pire la notion est considérée comme une élucubration de scientifiques fumeux, au mieux elle apparaît comme devant éternellement rester incomprise des non-initiés.

Le sens commun n'est pourtant peut-être pas si loin de la réalité des choses. Le terme de période de retour en hydrologie ressemble à ces mirages qui disparaissent au fur et à mesure que l'on s'en approche. Les raisons concrètes de cet évanouissement, c'est-à-dire de l'impossibilité présente d'avoir une définition véritablement scientifique, sont détaillées largement dans la présentation de M. Desbordes.

Plus généralement, il faut remarquer que l'hypothèse de stationnarité du phénomène n'est pas vérifiée, principalement à cause de l'influence des activités anthropiques. En particulier, les modes d'occupation des sols peuvent avoir des conséquences importantes sur l'hydrologie des bassins versants, que ce soit l'urbanisation responsable de l'imperméabilisation qui empêche l'eau de s'infiltrer, ou la déforestation et les nouvelles pratiques culturales qui peuvent entraîner une diminution des temps de concentration.

En dehors de ces changements qui modifient les valeurs de débit et de volume produits, les évolutions climatiques dues à ce qu'on l'on appelle le "global change », pourraient aussi avoir un impact sur le cycle de l'eau. Récemment, des simulations auraient montré qu'il fallait s'attendre à une augmentation de la lame d'eau moyenne annuelle qui tombe sur l'Europe.

Il semble clair aujourd'hui que les aléas à l'origine des inondations ne peuvent plus être considérés uniquement comme des phénomènes purement naturels, mais doivent intégrer dans leur définition, l'effet des activités humaines. Pour arriver à bien les comprendre, il faudrait utiliser simultanément les sciences naturelles pour analyser les mécanismes physiques, et les sciences sociales pour tenter d'évaluer l'influence du fonctionnement de nos sociétés.

Ce type de démarche semble encore plus pertinent pour approfondir la notion de vulnérabilité. Deux éléments sont à prendre en considération. La détermination des enjeux de la lutte contre les inondations, c'est-à-dire la question de savoir quels sont les ouvrages et les activités qu'il est important de protéger, permet de commencer à pouvoir quantifier les dommages. Il s'agit ici principalement des dommages tangibles directs. Les dommages intangibles ou indirects sont quant à eux difficilement appréciables.

A cette vue statique, il faut ajouter la prise en compte de la dynamique des évolutions. Ainsi, la responsabilité de l'augmentation des dommages peut aussi être attribuée à une occupation inconsidérée des lits mineurs au cours du temps.

Le deuxième volet de la définition de la vulnérabilité concerne les ressources disponibles. Ce sont elles qui vont déterminer la gamme des solutions réalistes.

Nous pouvons conclure que, dans le domaine de la lutte contre les inondations, l'analyse de l'aléa et de la vulnérabilité doit faire intervenir le social. La gestion du risque peut alors conduire des hydrologues à intervenir dans les processus de décision politique, au risque d'y perdre leur légitimité.

\section{III — LES POLLUTIONS}

Si toutes les activités anthropiques génèrent naturellement de la pollution, l'impact particulier de la ville provient de la concentration de ces activités. Celles-ci se déclinent de plusieurs façons : concentration des rejets domestiques, concentration des activités industrielles, concentration des activités publiques, et concentration des acteurs sociaux qui compliquent les processus de décision. L'utilisation d'une approche de gestion des risques va le montrer plus précisément.

La définition de l'aléa se révèle beaucoup moins simple que dans le cas des inondations. Il faut en effet tenir compte de plusieurs caractéristiques spécifiques.

Le nombre de paramètres responsables de ce que l'on appelle la pollution de l'eau tend à augmenter. A la suite des Agences de l'eau, on a commencé par s'intéresser aux matières oxydables (MO) et aux matières en suspension (MES). Au fil du temps, sont venus s'ajouter à la liste des substances à contrôler les micro-polluants, puis les nutriments.

L'impact des pollutions, en particulier sur les écosystèmes, peut être très diversifié. On distingue couramment les effets de choc dus à des rejets limités dans le temps, comme les rejets urbains de temps de pluie, les effets de stress qui caractérisent les atteintes produites par la répétition des pollutions qui, chacune prise isolément, ne sont pas catastrophiques, et les effets chroniques qui sont des impacts qui se réalisent au bout d'une longue période. Selon que l'on considère l'un ou l'autre de ces effets, la période de retour choisie pour l'événement dont on veut se protéger sera différente.

De manière encore plus générale, il faudra distinguer entre les effets à court terme et les effets à long terme. Ces derniers pourront être produits, par exemple, par des métaux lourds, piégés dans un premier temps dans les sédiments d'une rivière, et relégués peu à peu dans l'eau du fait des modifications géochimiques du milieu.

Enfin, parmi les aléas, il faut aussi compter ceux qui sont dus à des causes purement anthropiques. Il s'agira alors d'accidents, du type accident de transports de produits dangereux, ou lessivage de dépôts toxiques. Dans ce cas aussi, les statistiques sont difficiles à utiliser.

Comme pour les aléas, la définition de la vulnérabilité s'avère malaisée. Trois types de vulnérabilité peuvent être envisagés. La première peut être appelée physico-chimique. La pollution va entraîner un changement dans la composition physico-chimique du milieu, ce qui va avoir pour conséquence l'impossibilité d'employer l'eau pendant plus ou moins longtemps pour l'un ou l'autre des usages à laquelle elle est habituellement destinée. Dans la mesure où ces 
usages sont parfaitement définis, comme l'alimentation en eau potable, il paraît possible de quantifier ce type de vulnérabilité

Il existe aussi une vulnérabilité écologique. La pollution atteint plus ou moins profondément les écosystèmes. Dans le cas d'une disparition d'espèces utiles directement pour l'homme, par exemple pour son alimentation, la quantification semble encore possible. En revanche, cela semble difficile voire impossible, dans le cas où le préjudice est intangible, que ce soit pour les humains vivant actuellement, ou pour les générations futures.

Enfin, il faudrait prendre en compte un risque que l'on pourrait appeler biologique, même si aujourd'hui, il n'existe aucun exemple réel qui puisse l'illustrer. Il ne paraît pas impossible qu'une substance déversée dans l'eau ait une action mutagène sur des micro-organismes, ce qui pourrait entraîner l'éclosion de nouvelles maladies difficilement mầtrisables. Sans jouer les Cassandre, rappelons que tous les ans ce sont plusieurs milliers de molécules nouvelles qui sont diffusées sur notre planète.

Que ce soit en termes d'aléas ou de vulnérabilités, l'analyse en gestion du risque des pollutions conduit apparemment soit à des impossibilités soit, au moins, à des difficultés.

\section{IV ÉLÉMENTS POUR UNE MISE EN CEUVRE D'UNE GESTION DES RIS- QUES}

Au-delà des difficultés qui viennent d'être évoquées ci-dessus, on peut essayer d'envisager les éléments qui apparâtraient essentiels, dans le contexte actuel, à la mise en place d'une gestion simultanée des risques. Celle-ci est évidemment tributaire des outils scientifiques et techniques existants et de la demande sociale.

Il est clair que, tant dans le domaine des inondations que dans le domaine des pollutions, la connaissance des phénomènes est largement insuffisante. L'insuffisance de séries chronologiques longues de mesures représente un facteur limitant qu'il sera difficile d'ignorer avant un certain temps.

En dépit de ce constat, il faut bien se protéger et savoir comment le faire. Un homme-clé peut permettre à l'ensemble des activités gestion du risque de se dérouler: l'expert. Les conditions dans lesquelles il va travailler sont essentielles.

Son rôle est normalement de faire l'état des connaissances à un moment donné sur le problème qui lui est soumis et de montrer comment ces connaissances peuvent être utilisées dans le cadre d'une décision politique concernant des aspects réglementaires, l'aménagement ou des équipements. Définir jusqu'où une approche scientifique dans le domaine du risque peut justifier une telle décision n'est jamais chose facile. L'expert peut alors être amené à aller au-delà de ce que normalement il peut apporter. Il sert alors de caution et perd la justification de son intervention.

Le contexte réglementaire constitue un deuxième élément. La France s'est dotée en 1992 d'une loi sur l'eau qui promeut une gestion équilibrée des ressources naturelles et des usages. Une négociation entre tous les acteurs est instaurée à travers la mise en place de documents spécifiques: Schémas Directeurs d'Aménagement et de Gestion des Eaux et Schémas d'Aménagement et de Gestion des Eaux. Il paraît clair que la gestion des risques doit être discutée dans ce cadre.

Un certain nombre de projets peuvent apparaître comme des solutions au problème de protection contre les risques. Il faut choisir entre ces projets et entre les diverses variantes d'un même projet. Des critères de choix doivent donc être bâtis qui, comme pour tout projet, auront non seulement des dimensions techniques, mais aussi des dimensions écono- miques et des dimensions d'appréciation de la demande sociale. En outre, ils doivent aussi servir à hiérarchiser entre les risques de pollution et les risques d'inondation.

Enfin, dernier élément paraissant essentiel, l'existence de procédures permettant la possibilité de prendre en compte les différents niveaux géographiques. Il peut, en effet, y avoir conflit de solutions selon les échelles auxquelles on peut se placer pour traiter de la gestion des risques. Le principe physique de l'écoulement de l'eau de l'amont vers l'aval en est le principal responsable. Pour en rester à des exemples simples, faire un bassin de retenue sur un bassin versant peut entraîner une aggravation des conditions hydrauliques à l'aval; des concentrations de paramètre de pollution peuvent être admises en un point d'un cours d'eau et pas à un autre point.

\section{LA DIVERSITÉ DES APPROCHES}

En fait, beaucoup d'approches sont possibles pour traiter de la gestion des risques. La plus évidente reste l'approche scientifique et technique. Elle reste indispensable mais il faut se poser la question de ses limitations vis-à-vis du problème abordé. Celui-ci est clairement pluridisciplinaire. C'est-à-dire que les chercheurs, tels qu'ils sont organisés aujourd'hui en France, à l'intérieur de découpages disciplinaires plus ou moins stricts, auront toujours du mal à bien l'appréhender. Cette approche restera donc toujours insuffisante.

L'existence d'une politique de gestion des risques est évidemment dépendante de la prise de conscience des élus qui en ressentent la nécessité et l'intègrent entièrement dans les politiques municipales qu'ils mettent en œuvre. Qu'on le regrette ou non, c'est rarement le cas aujourd'hui. Pour un maire qui décrète inconstructibles des zones soumises à un risque d'inondation important, combien d'autres qui, n'ayant d'autre horizon de réflexion que la date des prochaines élections, pensent qu'une telle politique est contre-productive vis-à-vis de leurs concitoyens.

D'aucuns ont avancé que la vulnérabilité des sociétés humaines aux risques naturels était d'autant plus grande qu'une certaine culture du risque avait complètement disparu. Les changements rapides des populations urbaines en sont, pour certains, la raison. Les nouveaux arrivés sur un site ne peuvent connaître les chemins empruntés par l'eau en cas d'événements extrêmes. La construction de bâtiments dans le lit des cadereaux à Nîmes en est l'illustration.

Autant cette affirmation apparaît probante pour ce qui concerne la protection contre les inondations, autant la notion de culture du risque vis-à-vis des pollutions semble relativement peu pertinente. La nouveauté de ce phénomène, due à l'introduction de substances créées par les activités industrielles de l'homme ces deux derniers siècles dans le milieu naturel, cadre mal avec la temporalité longue qui sous-tend le mot de culture. Il faudrait plutôt parler de formation à la culture du risque dans ce domaine, ce qui n'est pas quelque chose de simple.

Une autre approche classique, est l'approche réglementaire. Celle-ci est ressentie comme complémentaire de l'approche scientifique et technique. La cohérence entre les deux approches est cependant difficile à assurer. Généralement les lois et règlements sont en avance sur les possibilités techniques. Par exemple, la loi sur l'eau de 1964 liait les rejets à la qualité des eaux, ce qui était impossible à faire avec les outils existants à l'époque.

Dans un premier temps, ce décalage peut entraîner un accroissement des recherches dans le domaine scientifique, ce qui est plutôt une bonne chose. En revanche, si pour de multiples raisons, le décalage n'est pas comblé au bout d'un certain temps, alors des effets pervers peuvent apparaître. En particulier, le respect de la loi et des règlements n'est plus considéré comme essentiel. 
Enfin, dans la mesure où les incertitudes restent grandes, tant pour la connaissance des aléas que pour celle de la vulnérabilité, il faudrait peut-être appliquer un des principes environnementalistes mis en avant ces dernières années, le principe de précaution. L'application de ce dernier permettrait une réflexion salutaire sur la signification d'une gestion des risques dans le domaine de l'eau, même si des limitations économiques peuvent très vite mettre des bornes à son utilisation.

\section{VI $\square$ CONCLUSION}

Nous avons essayé de montrer à travers une grille d'analyse tirée du concept de gestion du risque, les grandes incertitudes qui existaient dans la connaissance des aléas naturels.
Par ailleurs, l'intervention sur les aléas dus aux activités anthropiques et sur la vulnérabilité des établissements humains n'est pas principalement dépendante de la connaissance scientifique et technique des phénomènes.

Dans ce contexte, certains chercheurs et techniciens ont essayé de présenter des outils d'analyse scientifique comme pouvant servir directement aux processus de décision. Ils se sont ainsi mis dans une position où, servant de caution aux décideurs, ils n'étaient plus en mesure de relativiser les connaissances qu'ils utilisaient.

Ceci apparaît aujourd'hui comme une erreur aussi bien pour les scientifiques que pour les décideurs. Il est temps de bien séparer les responsabilités de chacun. La gestion des risques doit être une préoccupation essentiellement politique. A cette condition, la recherche scientifique pourra apporter des éléments, au même titre que d'autres approches, permettant de mieux asseoir les décisions. 